\section{Weaning: Welcher Spontanatmungstest erhöht Extubationsrate?}

Subirà C et al. Effect of Pressure Support vs T-Piece Ventilation Strategies During Spontaneous Breathing Trials on Successful Extubation Among Patients Receiving Mechanical Ventilation: A Randomized Clinical Trial. JAMA 2019; 321: $2175-2182$

Regelmäßig durchgeführte Spontanatmungstests können das Weaning von Intensivpatienten erleichtern und zu höheren Extubationsraten führen. Dabei kommen 2 WeaningStrategien zum Einsatz, wobei Patienten unterschiedlich stark und lange beansprucht werden. Subirà und Team wollten wissen, welche Strategie sich günstiger auf den Extubationserfolg auswirkt, und haben die 2-stündige T-Stück-Beatmung mit der 30-minütigen Pressure-SupportBeatmung verglichen.

Bei beatmeten Intensivpatienten kann die Extubationsbereitschaft mithilfe von regelmäßig durchgeführten Spontanatmungstests geprüft werden. Das tägliche Screening ging dabei in klinischen Studien mit einer kürzeren Dauer der invasiven Beatmung und größeren Extubationserfolgen einher. Aktuell kommen vor allem 2 unterschiedliche Strategien zum Einsatz, die sich in Bezug auf Dauer und Beanspruchung der Patienten deutlich voneinander unterscheiden. So gibt es die Beatmung über ein T-Stück über 2 Stunden sowie die 30-minütige Beatmung im Pressure-Support-Modus. Welche dieser beiden Weaning-Strategien zu einem besseren Behandlungsergebnis führt, wird bis heute untersucht und diskutiert.

Subirà und Kollegen wollten im Rahmen einer prospektiven, randomisierten klinischen Studie beide Methoden miteinander vergleichen. Sie stellten zuvor die Hypothese auf, dass weniger beanspruchende Spontanatmungstests zu einem größeren Extubationserfolg und einer geringeren Rate an Reintubationen führen würden. Die Studie fand zwischen Januar 2016 und April 2017 auf 18 spanischen Intensivstation statt. Der Nachbe- obachtungszeitraum endete im Juli 2017. Geeignete Patienten erfüllten folgende Kriterien:

- Alter ab 18 Jahren,

- invasive Beatmung von mindestens 24 Stunden sowie

- Erfüllung von Weaning-Kriterien.

Im nächsten Schritt teilten die Forscher jeden Patienten 1 von 2 Interventionsgruppen zu:

- Gruppe 1: Spontanatmungstest mit 2-stündiger Beatmung über ein T-Stück

- Gruppe 2: Spontanatmungstest über 30 Minuten im PressureSupport-Modus

Als primären klinischen Endpunkt definierten die Studienautoren eine erfolgreiche Extubation bis 72 Stunden nach dem ersten Spontanatmungstest frei von mechanischer Beatmung. Die wichtigsten sekundären Endpunkte waren die Rate an Reintubationen, die Verweildauer auf Intensivstation und im Krankenhaus insgesamt sowie schließlich die Krankenhaus- und 90-Tages-Mortalität.

\section{Pressure-Support überlegen}

1153 Patienten wurden randomisiert, 1018 von ihnen schlossen die Studie ab. Sie waren im Durchschnitt 62,2 Jahre alt mit einer Standardabweichung von 15,7 Jahren, 37,1\% von ihnen weiblich. 473 Patienten (82,3\%) in Gruppe 2 mit Pressure-Support, aber nur 428 Patienten (74,0\%) in der T-Stück-Gruppe konnten erfolgreich extubiert werden. Dieser Gruppenunterschied war dabei hochsignifikant.

In Bezug auf die sekundären Endpunkte lag die Reintubationsrate bei Gruppe 2 bei $11,1 \%$ und bei Gruppe 1 bei 11,9\%. Die mediane Verweildauer auf Intensivstation betrug in der Pressure-SupportGruppe 9 Tage im Vergleich zu 10 Tagen bei Einsatz eines T-Stückes. Diese Unterschiede waren nicht signifikant. Die mediane Verweildauer im Krankenhaus konnte für beide Gruppen auf 24 Tage beziffert werden und war demnach vergleichbar.
Die Krankenhaussterblichkeit lag mit $14,9 \%$ in Gruppe 1 im Gegensatz zu $10,4 \%$ in Gruppe 2 deutlich höher. Auf Grundlage dieser Ergebnisse sehen Subirà und Kollegen ihre Eingangshypothese damit als belegt an und empfehlen für das Weaning von invasiv beatmeten Intensivpatienten die Verwendung von kürzeren und weniger anspruchsvollen Spontanatmungstests.

\section{FAZIT}

In dieser randomisierten klinischen Studie, mit über 100 beatmeten Intensivpatienten führte ein Spontanatmungstest mit 30-minütigem Pressure-Support im Vergleich zum 2-stündigen Test mit T-Stück zu einer deutlich höheren Extubationsrate. Die Autoren sehen durch diese Resultate ihre Eingangshypothese bestätigt, dass kürzere und weniger anspruchsvolle Spontanatmungstests das Weaning erleichtern können.

Dipl.-Psych. Annika Simon, Hannover 